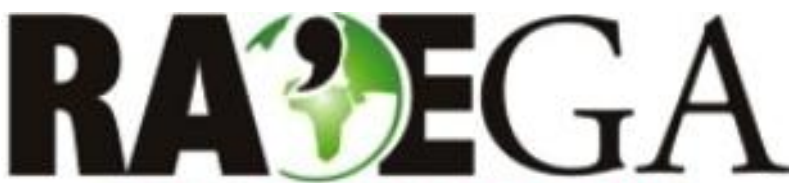

O ESPAÇO GEOGRÁFICO EM ANÁLISE

\title{
ANÁLISE MULTIFRACTAL DA VARIABILIDADE ESPACIAL PLUVIOMÉTRICA NO ESTADO DE MINAS GERAIS
}

\section{MULTIFRACTAL ANALYSIS OF PLUVIOMETRIC SPACE VARIABILITY IN THE MINAS GERAIS STATE}

\author{
Flavio Henrique Nery ${ }^{1}$, Maria Márcia Magela Machado²
}

\section{RESUMO}

A abordagem da geometria fractal permite identificar padrões dentro dos sistemas dinâmicos não-lineares, uma vez que os fractais são estruturas resultantes de processos caóticos. Assim, surge a dimensão fractal, para medir o grau de irregularidade, fragmentação e aspereza dos objetos. Diversos estudos apontaram a eficiência do cálculo fractal para compreensão da dinâmica das chuvas e sua distribuição espacial. Neste contexto, o Estado de Minas Gerais aparece como um arcabouço complexo de formadores das chuvas, desde a presença dos fatores atmosférico, como as massas de ar, até os elementos geográficos locais que influenciam diretamente na precipitação regional. Com isso, surge como questão as formas de análise do comportamento da distribuição espacial das chuvas e os benefícios da aplicação da abordagem fractal neste cenário que contém irregularidades pluviométricas em todas as regiões do território mineiro. Portanto, o objetivo deste trabalho foi compreender os diferentes níveis escalares da organização das chuvas em Minas Gerais, por meio do mapeamento de seus índices fractais e da análise de suas possíveis relações com o Zoneamento Climático de Minas Gerais. Através da análise e da espacialização do comportamento da precipitação foi observado um padrão no regime pluviométrico das regiões quando comparado com o Zoneamento Climático, apresentando maior pluviosidade no sul de Minas, devido, principalmente a influência dos sistemas atmosféricos e fatores locais. O método se mostrou eficaz na regionalização da precipitação, onde a dimensão fractal pode ser um indicador da distribuição das chuvas numa localidade.

Palavras chave: Geometria Fractal, Dimensão Fractal pluviométrica, Precipitação.

\section{ABSTRACT}

The fractal geometry approach allows identifying patterns within the nonlinear dynamic systems, since the fractals are structures resulting from chaotic processes. Thus, a fractal dimension appears to measure the degree of irregularity, fragmentation and roughness of the objects. Several studies showed the fractal fraction efficiency for the understanding of the rainfall dynamics and its spatial distribution. In this context, the State of Minas Gerais, Brazil, comes up with as a complex system of rain trainers, from the presence of atmospheric factors, such as air masses, to the geographic elements, sites that directly influence the regional precipitation. However, a question arises about the ways to analyze the behavior of the rainfall spatial distribution and the benefits of applying the fractal approach in this scenario, which contains rainfall irregularities in all regions of the territory of Minas Gerais. Therefore, the objective of this paper was to understand the various levels of the rainfall organization in Minas Gerais, by the mapping of its fractal indices and the analysis of its possible relations with the Climatic Zoning of Minas Gerais. Through the analysis and specialization of the precipitation behavior, it was observed a rainfall pattern in the regions, when compared to the climatic zoning, presenting higher rainfall in the south of Minas, mainly due to the influence of atmospheric systems and local factors. The method proved to be effective in the regionalization of precipitation, where a fractal dimension can be an indicator of the distribution of rainfall in a locality.

Keywords: Fractal Geometry, Fractal Dimension Rainfall, Precipitation.

Recebido em: 26/06/2017

Aceito em: 29/09/2018

\footnotetext{
${ }^{1}$ Universidade Federal de Minas Gerais, Belo Horizonte/AM, email: fhnery@gmail.com

2 Universidade Federal de Minas Gerais, Belo Horizonte/AM, email: mmarciamm@gmail.com
} 


\section{ANÁLISE MULTIFRACTUAL DA VARIABILIDADE ESPACIAL PLUVIOMÉTRICA NO ESTADO DE MINAS GERAIS}

\section{INTRODUÇÃO}

A compreensão da oscilação pluviométrica num determinado local desempenha um importante papel nas tomadas de decisão concernentes ao planejamento urbano e rural, afetando diretamente diversos setores sociais e econômicos. Entretanto, o estudo da precipitação se mostra como algo complexo, uma vez que os mecanismos dinâmicos que produzem a chuva variam em escalas temporais e espaciais, possibilitando a compreensão do processo somente após a ocorrência do episódio. Neste sentido, uma forma de buscar o entendimento deste fenômeno é o estudo da sua variabilidade, ou seja, análise dos registros efetuados num determinado período (séries temporais). 0 desafio que se coloca é o reconhecimento, com um grau mínimo de confiabilidade, da distribuição dos registros ao longo do tempo e a compreensão dos mecanismos que os definem.

$\mathrm{O}$ arcabouço de elementos formadores de chuvas é diverso, desde a presença dos fatores de escala sinótica, como as massas de ar, até os elementos geográficos locais de mesoescala, como as chuvas orográficas. Assim, surgem como questão as formas de análise do comportamento da distribuição espacial das chuvas e os benefícios da aplicação da abordagem fractal neste cenário que apresenta variações dos padrões pluviométricos ao longo de um determinado território.

Monteiro (1971) teve papel importante na busca de um método que melhor contemplasse à variação dos elementos do clima e os tipos de tempo, pois ele entendia que somente aplicação da estatística descritiva não seria suficiente para identificar todos padrões e suas relações. Com isso, nasce o conceito de ritmo e, em específico, os gráficos de análise rítmica. Nele são representadas, ao mesmo tempo, as variações diárias (ou horárias) dos elementos do clima e a sucessão diárias (ou horárias) dos estados atmosféricos, numa associação genética. Para construir os gráficos de análise rítmica, eles devem representar a sucessão dos estados atmosféricos sobre um lugar, seja a sucessão habitual ou aquelas tidas como sucessões extremas, definindo assim "anos padrões" (ZAVATTINI; BOIN, 2013).

Pereira e Christofoletti (2003) estudaram a distribuição espacial das chuvas no Estado de São Paulo utilizando o método de análise fractal e compararam com o trabalho de Monteiro (1973) sobre a descrição dos climas do estado de São Paulo, pautada sobretudo na análise rítmica pluviométrica. Comprovou-se aplicável a dimensão fractal como parâmetro quantitativo para a análise rítmica.

Neste contexto, surge a abordagem da geometria fractal como procedimento para pesquisa dos fenômenos climáticos, pois devido a sua regularidade escalar, as séries temporais podem apresentar padrões em suas estruturas e semelhanças nas dimensões fractais. Visando contribuir para o aprimoramento das discussões sobre a precipitação em Minas Gerais e apoiado conceitual e metodologicamente na geometria fractal, o objetivo geral desse trabalho é compreender os diferentes níveis escalares da organização das chuvas em Minas Gerais, por meio do mapeamento de seus índices fractais. Especificamente isso inclui:

- Espacializar a dimensão fractal da série histórica selecionada de Minas Gerais;

- Apontar o seu comportamento pluviométrico por meio da regionalização da chuva.

\section{SISTEMAS COMPLEXOS E GEOMETRIA FRACTAL}

Os procedimentos metodológicos adotados nos estudos dos fenômenos ambientais e geográficos tem uma relação direta com o maior embasamento tecnológico disponível atualmente nas várias áreas do conhecimento, ocorrendo melhorias em diversas pesquisas, favorecendo, assim, aperfeiçoamento nas coletas dos dados, tratamento e análise. Isso permitiu aprimorar os estudos no campo dos sistemas dinâmicos ou caóticos, considerando a complexidade dos sistemas e os estudos das suas partes componentes (CHRISTOFOLETTI, 1999). 


\section{ANÁLISE MULTIFRACTUAL DA VARIABILIDADE ESPACIAL PLUVIOMÉTRICA NO ESTADO DE MINAS GERAIS}

Neste sentido, a abordagem sistêmica aparece como alternativa ou complemento ao pensamento cartesiano. Esse método surge como uma alternativa, pois essa nova abordagem não veio com o intuito de destituir tudo o que existia a respeito de métodos de investigação da ciência, mas para agrupá-los e deles buscar uma maior compreensão da realidade (LIMBERGER, 2006).

No intuito de melhor compreender as diversidades de elementos, encadeamentos, interações e fluxos, o estudo da complexidade vem sendo considerado o principal agente moldador, reformulando e ultrapassando a concepção mecanicista e linear dos sistemas. 0 raciocínio básico se encontra na concepção de que a maior da natureza não é linear, comportando-se como sistemas dinâmicos e caóticos (VITTE; Guerra, 2004). Pela tradicional Geometria Euclidiana, muitas estruturas existentes na própria natureza não possuem dimensões bem definidas e quantificáveis. Tais formas são geralmente originadas de uma dinâmica caótica, imprevisível, aleatória (CHRISTOFOLETTI, 1997).

Lorenz (1996), ao repetir um modelo de previsão do tempo com sistemas de equações puramente simples e repetitivas, ele inseriu os mesmos dados de uma experiência realizada anteriormente, porém acidentalmente arredondados (a anterior possuía seis casas após a vírgula; esta, somente três). O novo gráfico gerado revelou uma curva semelhante à anterior no início, mas extremamente divergente ao longo de seu desenvolvimento posterior (Fig. 1). Isso foi chamado por Lorenz (1996) de "dependência sensível das condições iniciais" ou "Efeito Borboleta". Assim, a previsão do tempo a longo prazo seria inviável, pois os erros e as incertezas se multiplicam, formando um efeito de cascata ascendente através de uma cadeia de aspectos turbulentos, que vão dos demônios da poeira e tormentas até redemoinhos continentais que só os satélites conseguem ver (GLEICK, 1990).

Lorenz percebeu que essa aleatoriedade continha ordem, pois era produzida a partir de um sistema determinístico simples. Restava compreender como essa ordem se expressa matematicamente em cada caso, pois nem tudo na natureza ocorre de maneira caótica, irregular. Desse modo, criou-se uma ciência - ainda de acordo com Guerrini (1996): a da Dinâmica NãoLinear, que engloba o estudo do caos determinístico. Isso porque este é relacionado aos fenômenos não-lineares - regidos por equações com potências de números não-inteiros na variável independente.

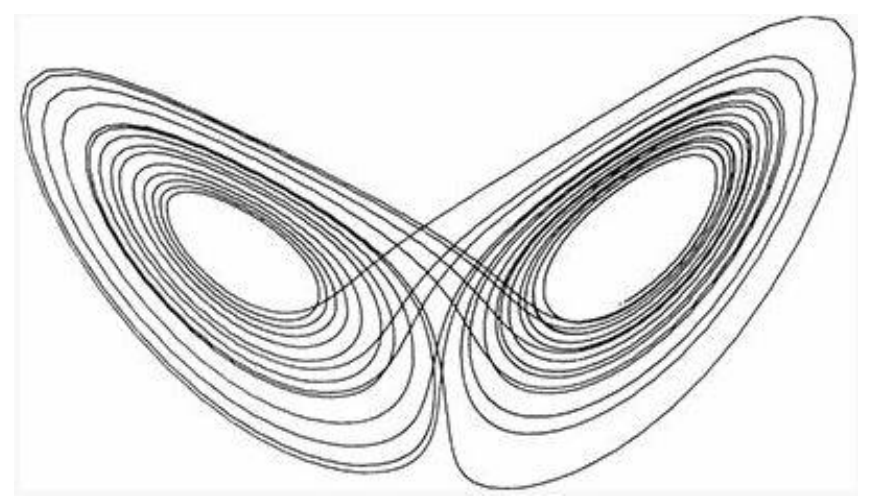

Figura 1 - Atrator de Lorenz: trajetórias giram, de maneira aparentemente aleatória, em torno de dois lobos. Fonte: Stewart, 1991.

Os fractais são expressões geométricas para o caos determinístico, ou seja, são formas estruturais resultantes de processos caóticos. Benoit Mandelbrot, matemático polonês radicado na França, ao longo dos anos 60, identificou as formas irregulares graças ao grande acesso a recursos computacionais, ele era funcionário da IBM, e recebeu a demanda de resolver os ruídos nas linhas telefônicas usadas para a transmissão de informações de um 


\section{ANÁLISE MULTIFRACTUAL DA VARIABILIDADE ESPACIAL PLUVIOMÉTRICA NO ESTADO DE MINAS GERAIS}

computador para outro dentro da empresa (PEREIRA; CHRISTOFOLETTI, 2003). Ao aumentar a escala de observação, se notava períodos com erros e períodos sem erros no momento da transmissão dos dados. Ao analisar somente as séries com erro ou sem erro, num exame mais apurado, tais séries com erros continham em si também períodos sem erros, ou seja, não havia um período continuo sem erro ou com erro. Este processo fez Mandelbrot (1983 apud GUERRINI, 1996) se lembrar de uma construção geométrica dentro da Matemática criada por Georg Cantor no século XIX e chamada de "barra triádica Cantoriana" ou "poeira de Cantor" (Fig. 2). Ela consiste de uma linha reta da qual se retira o terço médio; em seguida, se corta o terço médio dos segmentos restantes, e assim sucessivamente, até se gerar uma poeira de pontos infinitamente dispersos. Os erros de transmissão seriam, para Mandelbrot, "um conjunto de Cantor disposto no tempo" (PEREIRA; CHRISTOFOLETTI, 2003).

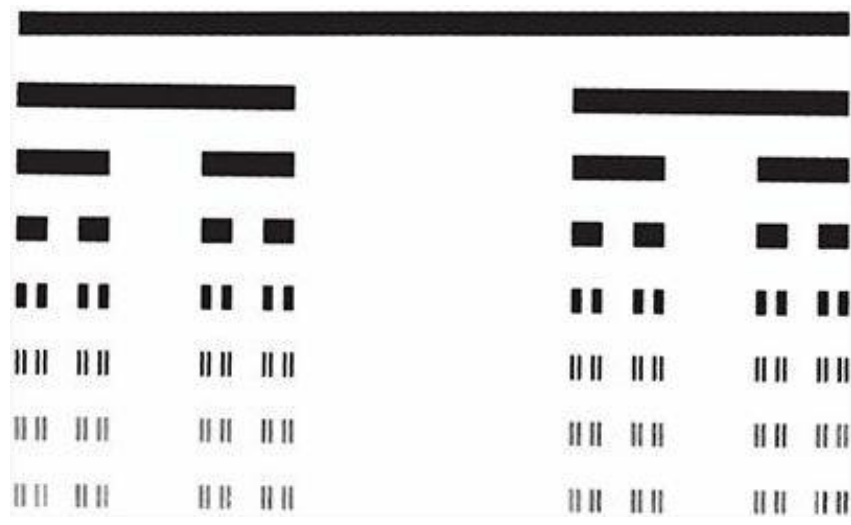

Figura 2 - Barra Triádica Cantoriana. Fonte: Stewart, 1991.

Portanto, figuras geradas pela repetição de processos simples passaram a representar melhor a complexidade da natureza, em relação à tradicional Geometria Euclidiana de linhas, planos, círculos, esferas, triângulos, cones e outras figuras "perfeitas" (PEREIRA; CHRISTOFOLETTI, 2003).

Para a geometria clássica, existem apenas três dimensões - isto é, três números especificam um ponto e as figuras possuem dimensões "inteiras": a dimensão de um ponto é zero, de uma reta é 1 , de um plano é 2 , e de uma figura volumétrica (como um cubo, por exemplo) é 3. No entanto, existem objetos que, dependendo da distância em relação ao observador, assumem diferentes dimensões. Gleick (1990) cita o exemplo do rolo de barbante, que, visto de muito longe parece um ponto e tem dimensão zero; numa proximidade maior, quando o rolo é percebido, passa a ter três dimensões; o barbante, por si só, é uma linha de dimensão 1; microscopicamente, ele passa a ser uma rede densa de fibras com dimensão 3; cada fibra, por si própria, tem dimensão 1, e assim por diante.

Percebendo que havia faixas de transição entre cada uma dessas dimensões, Mandelbrot (1983) criou um novo conceito para medir o grau de aspereza ou fragmentação, ou ainda, de irregularidade de um objeto: a dimensão fractal. Assim, um litoral recortado seria imensurável em sua extensão, mas possui um grau de rugosidade que o caracteriza e que permanece em diferentes escalas. Lam e De Cola (1993) mostram que "muitas variáveis ambientais são fractais e que o exame da dimensão fractal é útil para separar escalas de variação que possam ser o resultado de processos naturais". Existem várias maneiras de se calcular a dimensão fractal de um objeto (Fig. 3): a utilizada na elaboração deste trabalho será explicitada posteriormente.

De acordo com Christofoletti (1997) a dimensão fractal é "o valor do expoente do escalante relacionando o número de ocorrências desses elementos com a categoria dos seus diversos tamanhos". Partindo desse conceito, o 


\section{ANÁLISE MULTIFRACTUAL DA VARIABILIDADE ESPACIAL PLUVIOMÉTRICA NO ESTADO DE MINAS GERAIS}

autor considera que a dimensão fractal “ $D$ " entre 0 e 0,99 tem correspondência com estruturas baseadas em pontos como, por exemplo, os dados de precipitação. Sendo que, se a dimensão $D$ é igual a zero, temos um ponto, e, se $0<D<1$, temos uma poeira de pontos. Já os valores fractais entre 1,0 e 1,99 estão relacionados às estruturas espaciais lineares, como linhas costeiras e rios. Se $D=1$ a linha é reta e não fragmentada, denominada ideal, os outros valores intermediários se referem a estruturas que são mais do que uma reta, mas ainda não são um plano. As dimensões fractais entre 2,0 e 2,99 representam estruturas bidimensionais que se aplicam, por exemplo, à análise do formato de bacias hidrográficas, na modelagem digital de terrenos e os valores entre 3,0 e 3,99 correspondem a estruturas espaciais de representação volumétrica de uma categoria de ocorrência no interior de outro conjunto volumétrico, como o cálculo de reservas em jazidas minerais (VITTE; GUERRA, 2007).
Portanto, os fractais possuem dois componentes intrínsecos: a aleatoriedade, atributo que, segundo Christofoletti (1997), "encontra-se relacionado com a dinâmica caótica dos sistemas, assinalando a imprevisibilidade da forma específica a ser gerada" e; a regularidade, para Lam e De Cola (1993), é o relacionamento escalar regular entre o número de elementos e suas medidas é uma característica válido somente para "fractais ideais", chamando as estruturas que não atendem este requisito de "fractais com dependência de escala". Por exemplo, a representação de um lago não seria um fractal ideal, pois à medida que se aumenta a resolução escalar (até níveis milimétricos), a área representada aumentaria tanto que o mundo todo estaria debaixo d'água. Estes autores são rígidos com relação às proporções, que devem ser regulares em cada escala para se ter um fractal ideal. 


\section{ANÁLISE MULTIFRACTUAL DA VARIABILIDADE ESPACIAL PLUVIOMÉTRICA NO ESTADO DE MINAS GERAIS}

\begin{tabular}{|c|c|c|}
\hline Method & Algorithm & Estimate of $D$ \\
\hline $\begin{array}{l}\text { Stream number- } \\
\text { stream length }\end{array}$ & $\begin{array}{l}D=\log R_{b} / \log R_{1} \\
R_{b}=\text { bifurcation ratio } \\
R_{1}=\text { stream length ratio }\end{array}$ & \\
\hline \multirow{2}{*}{$\begin{array}{l}\text { Isarithm } \\
\text { (line-divider) }\end{array}$} & $L(\lambda)=k \lambda^{1-D}$ & Plot $\log L$ against $\log \lambda$ \\
\hline & $\begin{array}{l}\lambda-\text { step size } \\
L(\lambda) \text { - total length }\end{array}$ & $D=1-B$ \\
\hline \multirow[t]{2}{*}{ Variogram } & $2 \gamma(d)=k d^{d-2 \mathrm{D}}$ & Plot $\log \gamma(d)$ against log $d$ \\
\hline & $\begin{array}{l}d \text {-sampling interval } \\
2 \gamma(d) \text { - incremental } \\
\text { variogram }\end{array}$ & $\begin{array}{l}D=2-B / 2 \text { for profiles } \\
D=3-B / 2 \text { for surfaces }\end{array}$ \\
\hline Box-counting & $\begin{array}{l}N=k T^{-0} \\
1-\text { cell size } \\
N \text {-average } \\
\text { number of adjacencies }\end{array}$ & $\begin{array}{l}\text { Plot } \log N \text { against } \log I \\
D=-B\end{array}$ \\
\hline \multirow[t]{2}{*}{ Area-perimeter } & $A=k P^{210}$ & Plot $\log A$ against $\log P$ \\
\hline & $\begin{array}{l}\text { A-estimated area } \\
P \text { - estimated perimeter }\end{array}$ & $D=2 / B$ \\
\hline Power spectrum & $\begin{array}{l}P(\omega)=\omega^{2 D-5} \\
\omega-\text { frequency } \\
P(\omega)-\text { power }\end{array}$ & $\begin{array}{l}\text { Plot } \log P(\omega) \text { against } \log \omega \\
D=(5-B) / 2 \text { for profile } \\
D=(8-B) / 2 \text { for surface }\end{array}$ \\
\hline \multirow[t]{2}{*}{ Triangular prism } & & $\begin{array}{l}\text { Plot } \log A \text { against } \log \text { (step- } \\
\text { size }^{2} \text { ) }\end{array}$ \\
\hline & $\begin{array}{l}\lambda-\text { step size } \\
A(\lambda) \text { - total area }\end{array}$ & $D=2-B$ \\
\hline Size-frequency & $\begin{array}{l}N(A>a)=k a^{-D / 2} \\
N(A>a)-\text { no. of islands } \\
\text { above size } a\end{array}$ & $\begin{array}{l}\text { Plot } \log N /(A>a) \text { against } \\
\text { loga } \\
D=-2 B\end{array}$ \\
\hline
\end{tabular}

Figura 3 - Métodos para análise fractal. Fonte: Lovey; Schertzer e Tsonis, 1992.

A regularidade é representada por duas características fundamentais e correlatas dos fractais ideais: a autossimilaridade e a invariância escalar. Segundo Lam e De Cola (1993), a autossimilaridade é "a extensão para a qual o relacionamento entre o número de elementos constitutivos e suas medidas é verdadeiramente linear", o que leva a invariância escalar. Por esta característica, as estruturas fractais contêm cópias de si mesmas, ou seja, o padrão geométrico se repete nas diversas escalas de grandeza espacial ou de observação.

As características essenciais dos fractais para Guerrini (1996) são: "autossimilaridade, complexidade infinita e formação através de um processo de realimentação". Este autor se baseia na definição do matemático Dupont: "fractais são objetos que apresentam autossemelhança e complexidade infinita, ou seja, sempre contêm cópias, aproximadas ou não, de si mesmos, e são gerados pela interação de processos simples" (GUERRINI, 1996).

\section{DINÂMICA CLIMÁTICA DE MINAS GERAIS}

A variabilidade anual e interanual é um aspecto marcante da distribuição das chuvas no estado de Minas Gerais, com reflexos na agricultura, no abastecimento hídrico e energético. Devido à sua localização geográfica, Minas Gerais sofre a influência de fenômenos meteorológicos característicos de latitudes médias e tropicais que ditam a existência de duas estações bem distintas, sendo uma seca e a outra chuvosa (ABREU, 2008).

A complexidade da origem da precipitação no território mineiro pode ser compreendida nas ações dos sistemas atmosféricos, principalmente, os sistemas frontais ou Frente Polar Atlântica (FPA), as linhas de instabilidades tropical ou tropicais (IT), além 


\section{ANÁLISE MULTIFRACTUAL DA VARIABILIDADE ESPACIAL PLUVIOMÉTRICA NO ESTADO DE MINAS GERAIS}

do estabelecimento ocasional (durante a primavera e principalmente no verão) da Zona de Convergência de Umidade (ZCOU) e da Zona de Convergência do Atlântico Sul (ZCAS), resultando em chuvas durante vários dias (CAVALCANTI et al., 2009 e NIMER, 1989). Além destes, conforme Tarifa (1994), o efeito orográfico desempenha um importante papel no desenvolvimento de instabilidades convectivas locais (ICL), assumindo um fator de relevância em Minas Gerais. Segundo Nimer (1989), o estado mineiro sofre perturbações atmosféricas, principalmente, vindas de sul, de leste e de oeste. No Sul, temos as entradas da FPA e massa polar (MA); a leste os efeitos das ondas do Leste que, segundo o autor "são formações ondulatórias na média e alta atmosfera que se deslocam na região equatorial, de oeste para leste e a oeste as ações dos núcleos de baixa pressão (IT)".

As características dos climas, tanto na escala temporal como na escala espacial, são explicadas pelos elementos da circulação geral da atmosfera e a interação com sistemas atmosféricos de escala sinótica. A causa básica e fundamental do movimento atmosférico, horizontal ou vertical, é o desequilíbrio na radiação líquida, na umidade e no momentum entre as baixas e altas latitudes e entre a superfície da Terra e a atmosfera (LUCAS, 2007). Ayoade (2004) ainda destacou que a topografia, a distribuição das superfícies continentais e oceânicas e as correntes oceânicas constituem outros fatores que influenciam a circulação atmosférica.

De acordo com Reboita et al. (2010), o regime pluvial do Estado de Minas Gerais é afetado pela ocorrência de fenômenos atmosféricos importantes, que o caracterizam com um verão chuvoso (novembro-março) e um inverno seco (maio-setembro). Tais fenômenos consistem de chuvas frontais (frentes frias), chuvas convectivas e chuvas geradas pela influência da Zona de Convergência do Atlântico Sul (ZCAS).

Mello e Viola (2012), constataram que as maiores intensidades ocorrem nas regiões leste e noroeste de Minas Gerais, o que pode ser explicado pela maior influência da Zona de Convergência do Atlântico Sul, além de ocorrência de chuvas convectivas. Foi possível, também, constatar intensidades intermediárias nas regiões sul e central e os menores valores para as regiões norte e nordeste de Minas Gerais. Para maiores durações, se verificou, para a região sul, ocorrência de altas intensidades, o que está associado à entrada com maior frequência de frentes frias, produzindo chuvas de longa duração.

O governo do estado de Minas Gerais criou uma ferramenta para subsidiar políticas de desenvolvimento socioeconômico e ecológico de uma região, o Zoneamento Ecólogico-Econômico (ZEE). Uma das pastas do ZEE é o Zoneamento Climático, onde a delimitação das regiões climaticamente homogêneas permite, não só estabelecer os indicadores do potencial do meio físico e biótico para a região em estudo, mas também, juntamente com as delimitações das áreas homogêneas sob o ponto de vista socioeconômico, contribuir para o desenvolvimento sustentável da região.

Para gerar o mapa de classificação climática definidas pelo Conselho de Política Ambiental (COPAM), segundo o indicador climático de Thornthwaite (Fig. 4), foram utilizados os dados presentes nas Normais Climatológicas (1961-1990); registros mensais dos elementos climáticos de 39 estações climatológicas pertencentes ao Instituto Nacional de Meteorologia (INMET). Isto permitiu obter a informação de forma pontual detectando peculiaridades geográficas locais (SCOLFORO; CARVALHO; OLIVEIRA, 2008). 


\section{ANÁLISE MULTIFRACTUAL DA VARIABILIDADE ESPACIAL PLUVIOMÉTRICA NO ESTADO DE MINAS GERAIS}

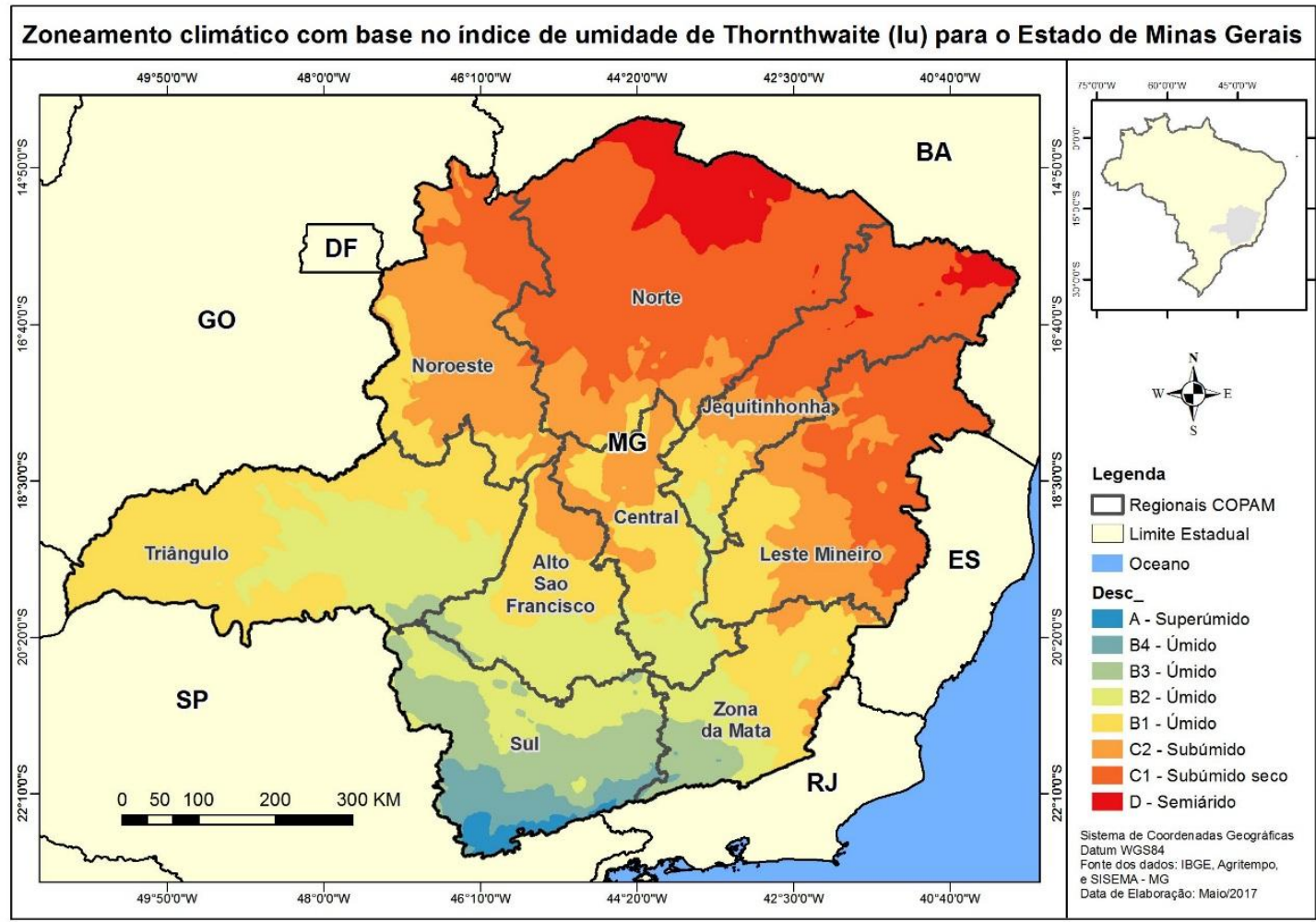

Figura 4 - Mapa do Zoneamento Climático de Minas Gerais. Fonte: Scolforo, Carvalho e Oliveira (2008).

\section{MATERIAS E MÉTODOS}

As séries históricas das estações pluviométricas foram obtidas no Sistema de Monitoramento Agrometeorológico (AGRITEMPO), onde são armazenadas informações coletadas de diversas empresas públicas e privadas. Neste estudo, analisamos os dados disponibilizados do Instituto Nacional de
Meteorologia (INMET) e a Companhia Energética de Minas Gerais (CEMIG). Também foram utilizados o limite estadual e do Brasil disponibilizado pelo Instituto Brasileiro de Geografia e Estatística (IBGE) e o limite das regionais utilizadas como referência pelo Sistema Estadual de Meio Ambiente e Recursos Hídricos (SISEMA) de Minas Gerais (Fig. 5). 


\section{ANÁLISE MULTIFRACTUAL DA VARIABILIDADE ESPACIAL PLUVIOMÉTRICA NO ESTADO DE MINAS GERAIS}

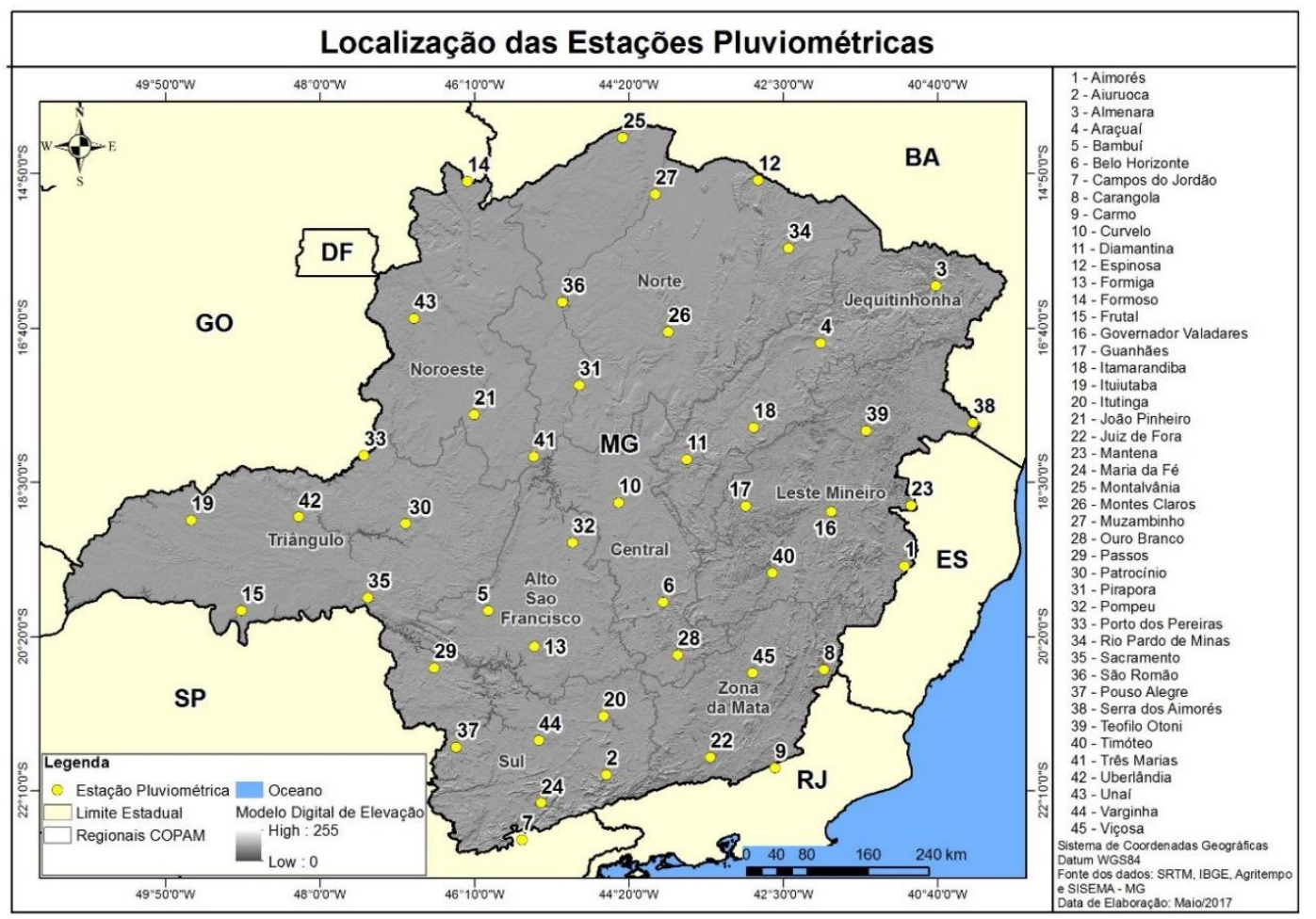

Figura 5 - Mapa de localização das estações pluviométricas selecionadas. Fonte: elaborado pelo autor.

Considerando a distribuição espacial das estações meteorológicas no Estado, o potencial de cobertura de cada estação em termos de área que de acordo com a orientação da WMO (WMO-No. 488) é, em média, $150 \mathrm{~km}$ e a existência da série histórica completa, foram selecionadas para este estudo 45 estações pluviométricas.

A metodologia utilizada neste estudo é uma adaptação daquela aplicada para análise da precipitação no estado de São Paulo por Christofoletti (1997), por sua vez baseada em Olsson et. al. (1992). Para cálculo da dimensão fractal é utilizado o procedimento de estimativa conhecido como Box Counting ou contagem de caixas. Este método consiste, basicamente, na contagem do número de ocorrências (frequência) de determinado volume de chuva (limiar estabelecido) num intervalo de tempo (segmento). Conforme MAYER (1992) e Lovejoy et. al. (1987) se trata então da contagem de ocorrências por segmento, ou seja, da relação entre a frequência de ocorrência de determinado volume de chuva por intervalo de tempo.
Segundo Christofoletti (1997), é necessário existir, dentro do período analisado dois anos atípicos, um com chuva acima da média e outro com chuva abaixo da média. Além disso, deve existir também um ano onde a precipitação tenha ficado dentro média histórica. A primeira etapa do trabalho consistiu, então, na validação do período pré-selecionado segundo esta condição imposta pela metodologia. 0 procedimento de verificação foi feito por amostragem. Foram selecionadas sete estações meteorológicas (Belo Horizonte, Campos do Jordão, Governador Valadares, Itapira, Montes Claros, Passos e Uberlândia) em função de sua localização geográfica e calculada a precipitação total anual para cada uma. Ao se comparar os valores obtidos com as médias das séries históricas fornecidas pelo AGRITEMPO, se constatou que a condição estava atendida, logo o período pré-selecionado foi efetivado como aquele de análise.

Christofoletti (1997) propôs a análise apenas dos dados de três semestres dentre aqueles considerados chuvosos no período 


\section{ANÁLISE MULTIFRACTUAL DA VARIABILIDADE ESPACIAL PLUVIOMÉTRICA NO ESTADO DE MINAS GERAIS}

analisado: o de maior volume de precipitação, o de menor volume e um com volume dentro da média histórica. Tendo como parâmetro que o semestre chuvoso em Minas Gerais corresponde aos meses de outubro a março, no período analisado o semestre de maior precipitação ocorreu entre os anos de 2008 e 2009, o mais seco entre os anos 2013 e 2014 e o semestre que teve o volume mais próximo da média histórica foi entre os anos 2012 e 2013.

O método de contagem de caixas investiga o comportamento escalar do fenômeno. 0 procedimento geral consiste na divisão sequencial do conjunto total de dados, diminuindo gradualmente o tamanho dos segmentos pela metade até chegar a uma unidade de tempo, e na contagem da frequência do volume de chuva em cada um deles para cada limiar estabelecido. Em função do processo de divisão do semestre chuvoso sucessivamente por dois, o comprimento original do segmento de 182 dias teve que ser ampliado para 192 dias, gerando as seguintes frações do segmento: 96 dias, 48 dias, 24 dias, 12 dias, 6 dias, 3 dias e 1 dia. Para definição dos valores limiares de quantidade de precipitação foi considerado como chuva valores a partir da taxa de acumulação maior ou igual a $1 \mathrm{~mm} /$ hora (BARRY; CHORLEY, 2013), diferentemente de Pereira e Christofoletti (2003) que consideraram como chuva os registros maiores ou iguais a $0,1 \mathrm{~mm} / \mathrm{dia}$. Foram definidos os seguintes valores como limiares: 1, 10, 20 e 40 $\mathrm{mm} /$ dia. Abaixo segue um exemplo da segmentação dos dias sua relação com o limiar definido em $5 \mathrm{~mm} /$ dia (Fig. 6).

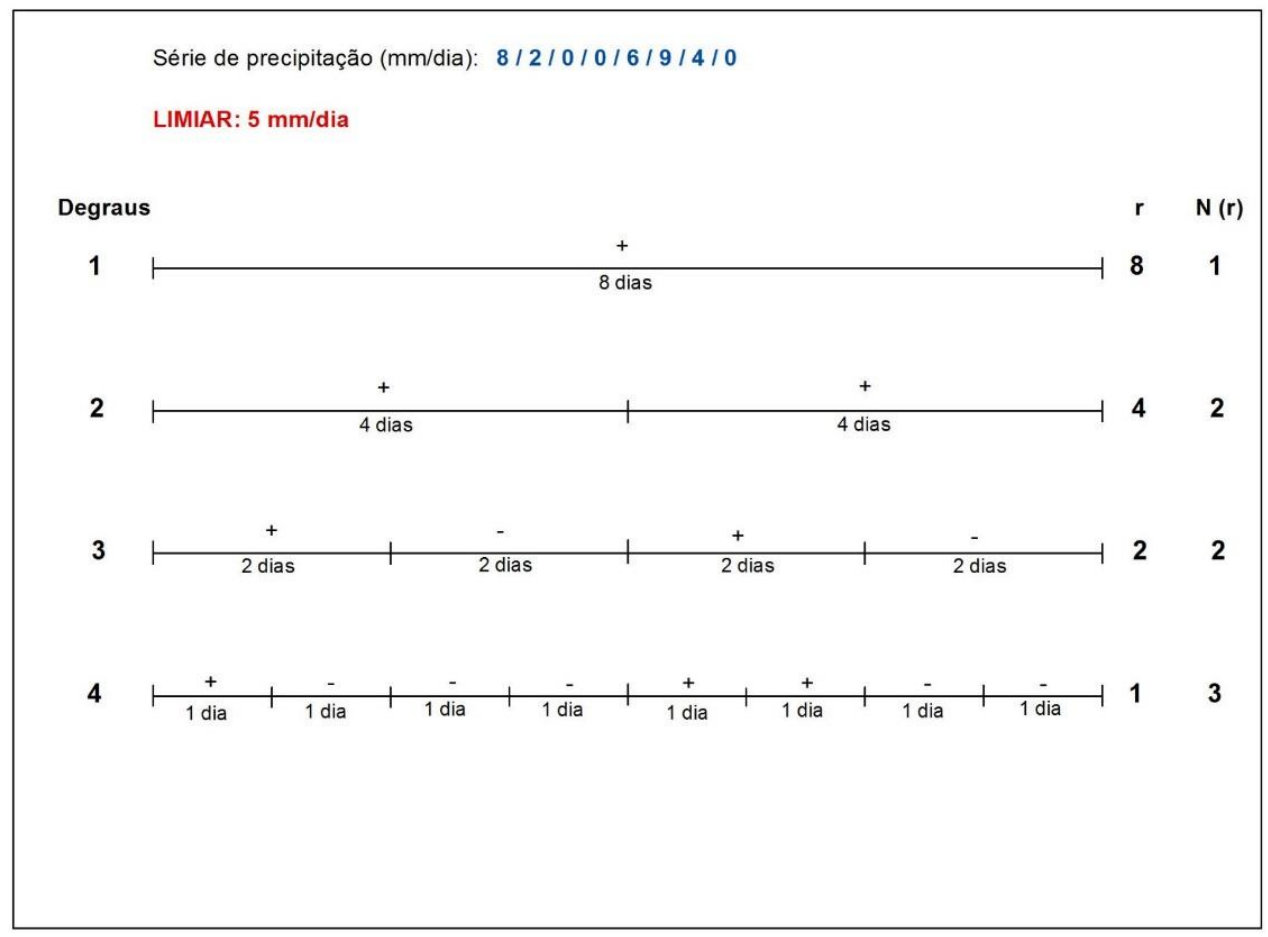

Figura 6- Exemplo da segmentação da série histórica para análise fractal. Fonte: elaborado pelo autor.

A contagem da frequência de ocorrência dos valores limiares de chuva no segmento original e em suas frações foi realizada no software Excel e permitiu a construção de gráficos em escala logarítmica de frequência versus segmento. Se a representação gráfica da relação dessas funções logarítmicas for uma reta, o evento possui dimensão fractal (PEREIRA; CHRISTOFOLETTI, 2003). O valor da dimensão fractal é o coeficiente angular do gráfico log box 


\section{ANÁLISE MULTIFRACTUAL DA VARIABILIDADE ESPACIAL PLUVIOMÉTRICA NO ESTADO DE MINAS GERAIS}

counting (frequência do limiar ( $N(\mathrm{r})$ )) versus - log box size (tamanho do segmento $(r)$ ).

Os índices fractais obtidos para cada uma das 45 estações, por limiar, foram inseridos em ambiente SIG (Sistema de Informação Geográfica) no software ArcGIS 10.4.1 e interpolados para gerar superfícies com o padrão de distribuição de chuva no estado de Minas Gerais em cada semestre analisado. Foram testados os interpoladores espaciais de krigagem ordinária (Ordinary Kriging-KRI) e de função de base radial (Radial Basis Functions - RBF). Como as superfícies geoestatísticas geradas por meio da krigagem ordinária apresentaram menor ocorrência de círculos concêntricos, estas foram selecionadas para apresentação dos resultados obtidos.

\section{RESULTADOS E DISCUSSÃO}

Realizou-se a análise fractal em busca de compreender a espacialização da chuva entre as regiões do Estado e suas peculiaridades locais. Existe uma variação entre os períodos e os limiares. O período chuvoso tende a maior regularidade no maior segmento, pois a ocorrência de chuva é mais comum de acontecer se comparado com os demais. O contrário acontece nos menores segmentos, uma vez que para ser preenchido, necessita de uma frequência maior de ocorrência. Os limiares também indicam a variação pluviométrica, onde o menor limiar tende a apresentar uma regularidade entre as estações, ao contrário do maior limiar que apresenta uma amplitude e dispersão maiores no segmento 1 , por exemplo. A localização também é um fator que altera a dinâmica pluviométricas, ainda mais em Minas Gerais onde temos diferentes regimes hídricos.

As ocorrências de chuvas dentro de cada segmento, onde a linha indica rupturas na frequência da precipitação, ou seja, quando surge uma reta entre o menor e os maiores números, significa que os segmentos correspondentes foram preenchidos pela frequência de chuva (PEREIRA; CHRISTOFOLETTI, 2003).

Os comportamentos das estações pluviométricas dentro do limiar $1 \mathrm{~mm}$, seguiram as premissas estabelecidas pelo Zoneamento Climático de Minas Gerais (SCOLFORO; CARVALHO; OLIVEIRA, 2008), onde cada região possui suas peculiaridades locais e influências atmosféricas.

A distribuição da precipitação nos mapas gerados demostra que o índice da dimensão fractal decaem com o aumento dos limiares. Isto ocorre devido ao limiar $1 \mathrm{~mm}$ contemplar qualquer registro de chuva acima ou igual a 1 $\mathrm{mm}$. Enquanto isso, a frequência reduz à medida que aumenta os limiares, pois a ocorrência se torna mais difícil. Outro fator que altera os valores das dimensões fractais são as diferenças entre os períodos chuvosos e secos, pois a frequência de chuvas tende a diminuir à medida que se direciona para o período mais seco.

$\mathrm{Na}$ análise dos mapas, os valores da dimensão fractal reduzem à medida que aumenta os limiares. Isso ocorre devido a relação de ocorrência dos eventos, onde o limiar $1 \mathrm{~mm}$ engloba todos os registros de chuvas de menor ou igual valor; seguindo a mesma premissa, o limiar $40 \mathrm{~mm}$ ocorre em frequências menores, por representar maiores volumes. Outro fator que tende a alterar os valores da dimensão fractal é o período selecionado que contempla semestres úmidos e secos. Ele reduz na estação mais seca em função da menor frequência de chuvas.

No período considerado chuvoso (08/09), o limiar $1 \mathrm{~mm}$ apresentou valores entre 0,76 e 0,91; o limiar $10 \mathrm{~mm}$ entre 0,59 a 0,78; o limiar $20 \mathrm{~mm}$ entre 0,42 a 0,64 e o limiar $40 \mathrm{~mm}$ entre 0 e 0,52 (isto significa que em alguns locais não houveram registros de chuvas nesta magnitude). A distribuição das chuvas pelo território mineiro apresentou uma regularidade nos limiares 1, 20 e $30 \mathrm{~mm}$, com faixas da dimensão fractal estendendo sentido Noroeste Sudeste. Ao Sul e parte da Zona da Mata apresentaram os maiores índices fractais, enquanto no extremo norte das regiões Norte e Jequitinhonha temos os valores menores. No limiar $40 \mathrm{~mm}$, a irregularidade nas ocorrências provocou uma discrepância na distribuição das chuvas, destacando-se os maiores valores na 


\section{ANÁLISE MULTIFRACTUAL DA VARIABILIDADE ESPACIAL PLUVIOMÉTRICA NO ESTADO DE MINAS GERAIS}

Zona da mata e parte do Leste mineiro. $\mathrm{Na}$ transição entre os limiares 1 a $40 \mathrm{~mm}$, acontece um deslocamento da faixa de maior valor fractal do sentido Sul para o Leste, caracterizando as regiões Zona da Mata e Leste com as maiores ocorrências de chuvas torrenciais (igual ou acima
de
40
$\mathrm{mm}$ )
(Fig.
7).

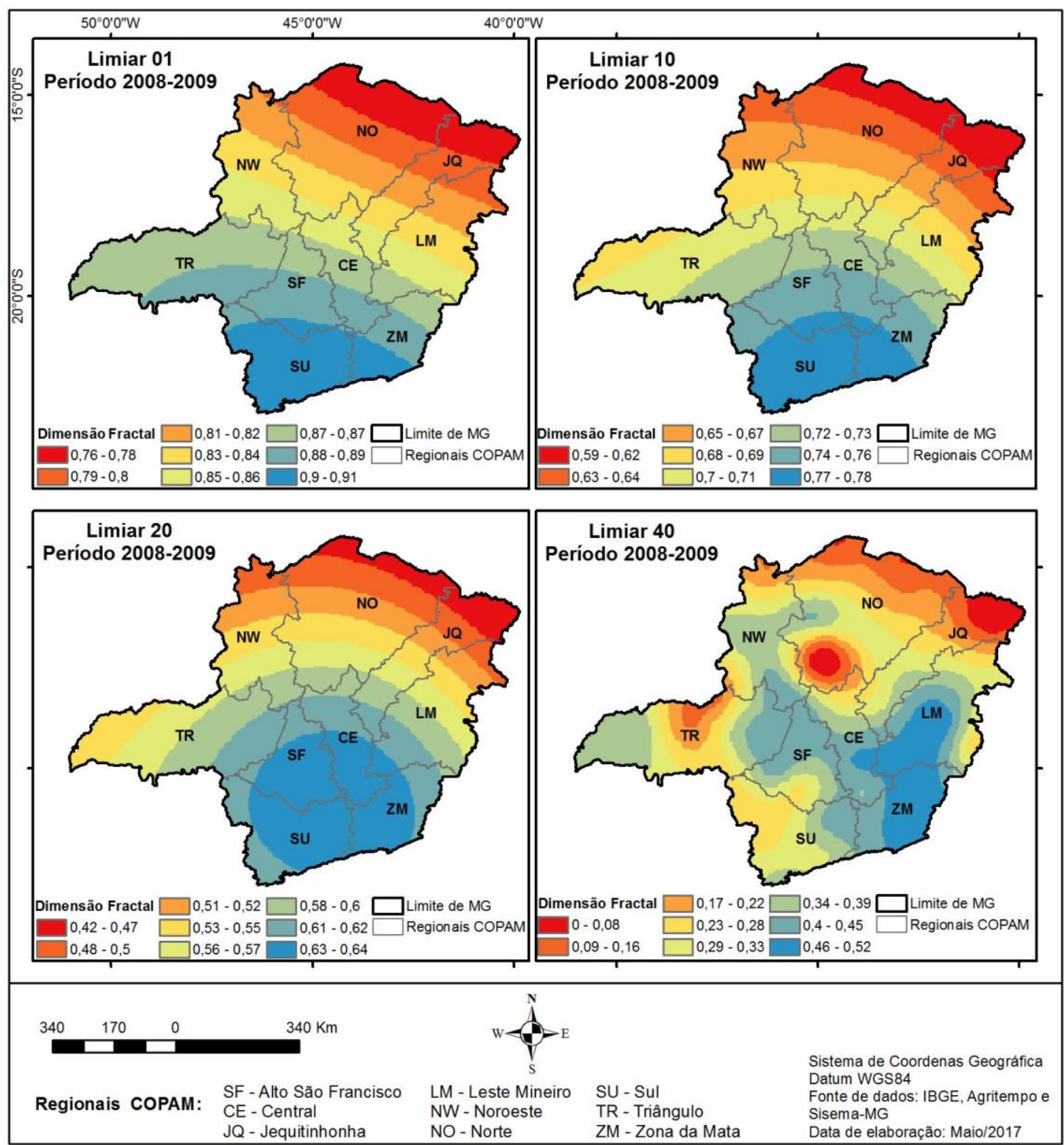

Figura 7 - Mapa da espacialização da chuva em Minas Gerais para o período 2008-2009.

No período considerado padrão (12/13), o limiar $1 \mathrm{~mm}$ apresentou valores entre 0,71 e 0,92; o limiar $10 \mathrm{~mm}$ entre 0,46 a 0,75; o limiar $20 \mathrm{~mm}$ entre 0,25 a 0,63 e o limiar $40 \mathrm{~mm}$ entre 0,12 e 0,32 . No limiar $1 \mathrm{~mm}$, se observa um padrão na distribuição da precipitação com faixas contínuas, onde a dimensão fractal reduz os valores no sentindo sul-norte. Já no limiar 10 e 20 $\mathrm{mm}$, as faixas estão descontinuas com destaque para as regiões Sul e Triângulo com os maiores índices. A região da Zona da Mata apresentou o maior valor no limiar 40 mm (Fig. 8). 


\section{ANÁLISE MULTIFRACTUAL DA VARIABILIDADE ESPACIAL PLUVIOMÉTRICA NO ESTADO DE MINAS GERAIS}

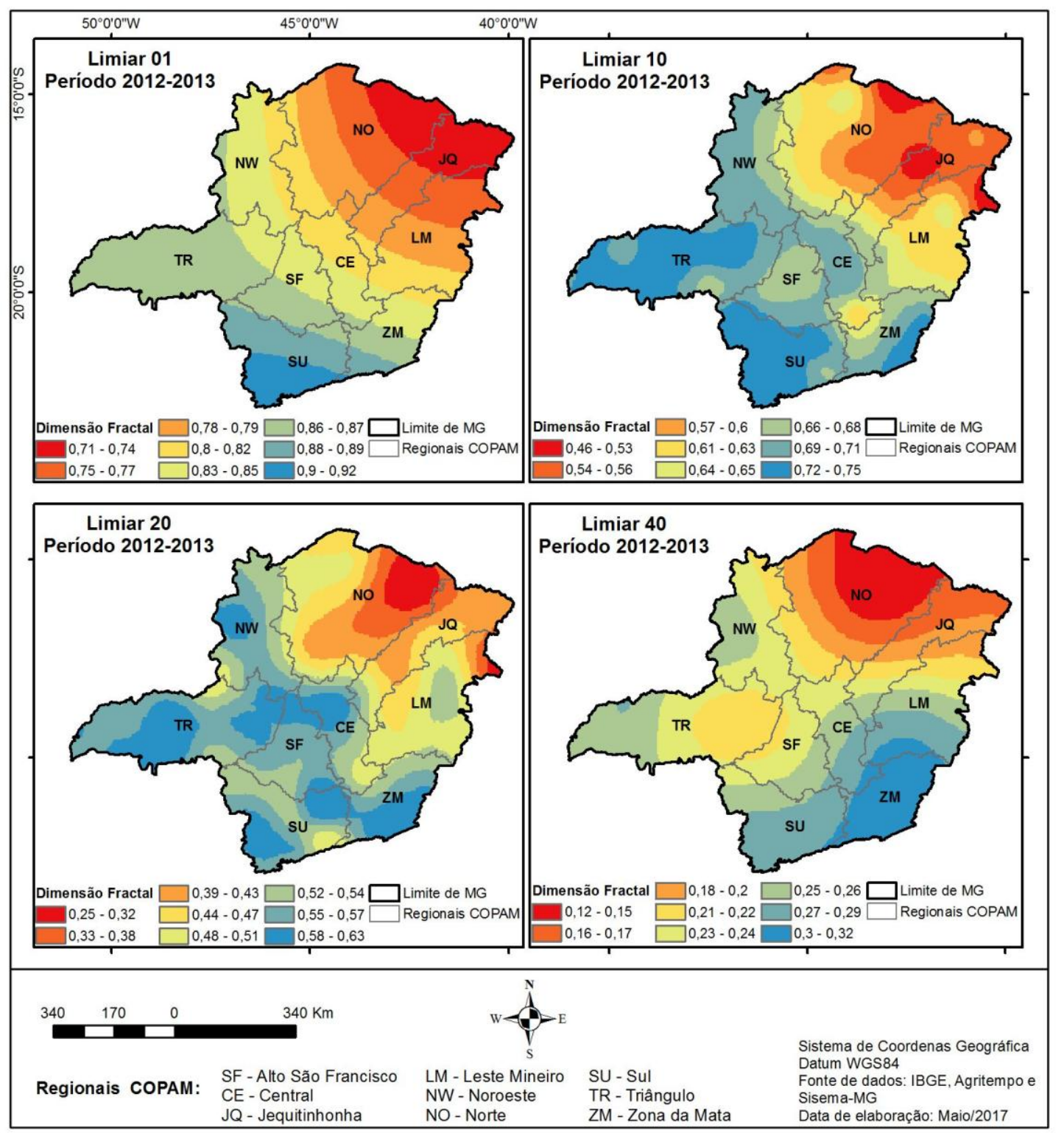

Figura 8 - Mapa da espacialização da chuva em Minas Gerais para o período 2012-2013.

No período considerado seco (13/14), o limiar $1 \mathrm{~mm}$ apresentou valores entre 0,75 e 0,88; o limiar $10 \mathrm{~mm}$ entre 0,53 a 0,72; o limiar $20 \mathrm{~mm}$ entre 0,39 a 0,58 e o limiar $40 \mathrm{~mm}$ entre 0,07 e 0,46 . Observa-se em todos os limiares a irregularidade entre as faixas de precipitação, devido ser este o período mais seco da série estudada. No limiar $1 \mathrm{~mm}$, os maiores índices estão nas regiões do Triângulo e Noroeste, com destaque para o Sul de Minas e Zona da Mata que demostram a influência da topografia para os altos valores fractais. Nos limiares seguintes as regiões com os maiores valores fractais são a Zona da Mata e Leste Mineiro (Fig. 9). 


\section{ANÁLISE MULTIFRACTUAL DA VARIABILIDADE ESPACIAL PLUVIOMÉTRICA NO ESTADO DE MINAS GERAIS}

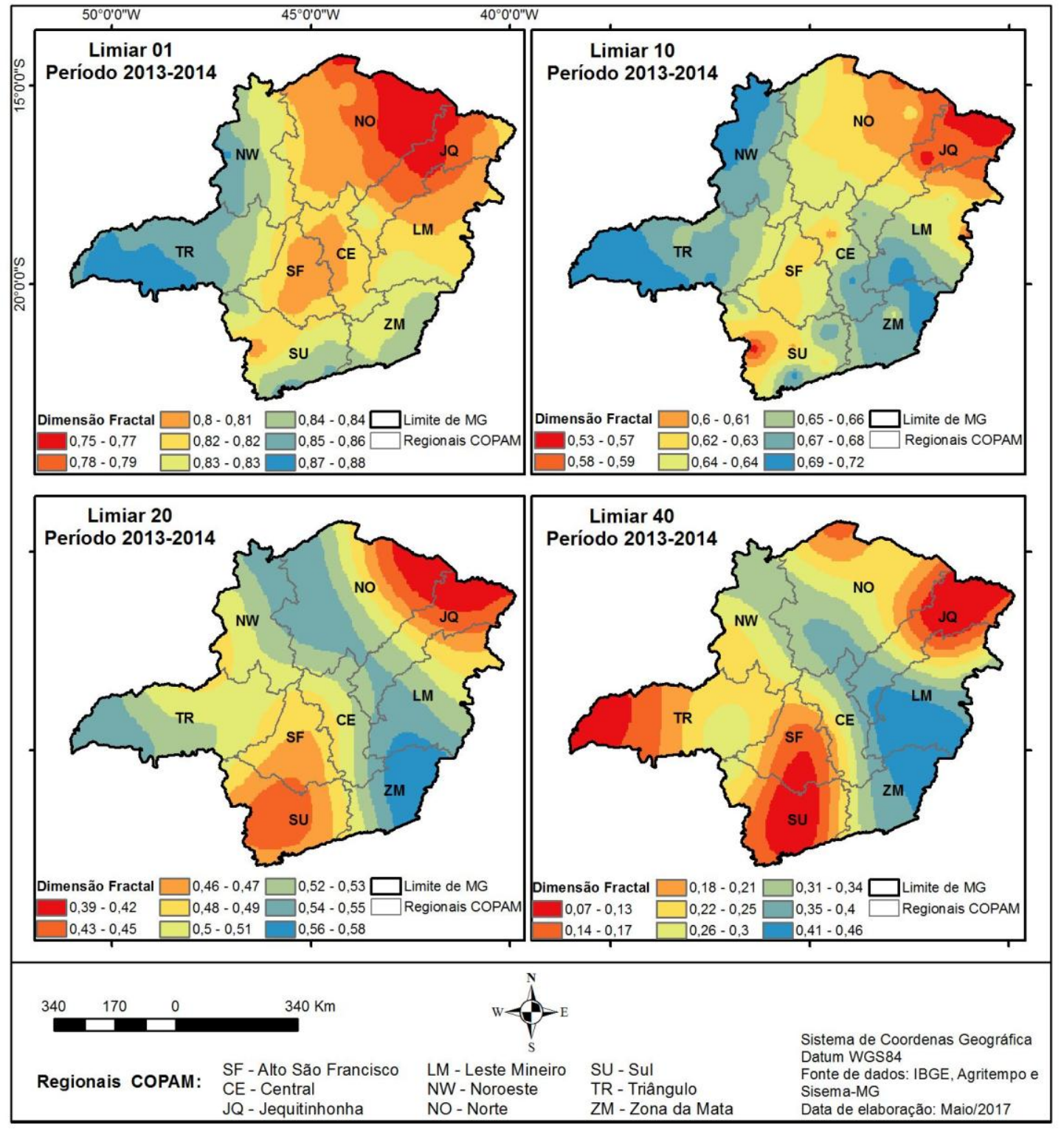

Figura 9 - Mapa da espacialização da chuva em Minas Gerais para o período 2013-2014.

Ao analisar a transição dos limiares dentro dos anos padrões é possível observar que a distribuição da pluviosidade é controlada pela disposição do relevo, pela atuação das frentes polares e pela continentalidade, conforme indicado por Lucas (2007) e AYOADE (2004). Ao observar a maior precipitação no sul do Estado, associamos com a presença das frentes polares que contribuem para uma maior frequência ao longo do semestre chuvoso. A influência da topografia local e as perturbações de oeste também contribuem para o alto valor da dimensão fractal nesta região. Este cenário altera à medida que deslocamos para o ano de "seca", pois o bloqueio atmosférico impede a entrada das massas polares no Estado, como já citado por Nimer (1989).

Já a ocorrência das ZCAS contribui para a precipitação nas regiões do Triângulo, Noroeste, Central e, principalmente, Zona da Mata e Leste Mineiro. Nestes casos se observa uma influência na concentração de chuva, onde a dimensão fractal apresentou valores maiores no ano de "seca". Um contribuinte para os elevados índices 


\section{ANÁLISE MULTIFRACTUAL DA VARIABILIDADE ESPACIAL PLUVIOMÉTRICA NO ESTADO DE MINAS GERAIS}

são os processos convectivos (MELLO; VIOLA, 2012).

Na porção norte que contemplam as regiões Norte e Jequitinhonha se percebe os menores índices fractais. O domínio de situações anticiclônicas contribuem para inibir o avanço dos sistemas mais úmidos (MELLO; VIOLA, 2012). As linhas de instabilidades tropicais constituem o principal fator genético das chuvas nestes locais (TARIFA, 2004), com influência discreta da frente polar.

Ao observar o Mapa do Zoneamento Climático de Minas Gerais (Fig. 4), a precipitação desloca com os maiores valores do Sul em direção ao Norte do Estado. Conseguimos enxergar este direcionamento apenas nos anos "chuvoso" e "padrão" no limiar $1 \mathrm{~mm}$. Nos outros limiares temos uma distribuição irregular nas regiões do Estado.

\section{CONSIDERAÇÕES FINAIS}

Neste trabalho, se buscou identificar o padrão pluviométrico na espacialização da dimensão fractal, por meio da relação entre os anos padrões e os seus limiares. 0 reconhecimento dos padrões regionais contribui para o planejamento governamental. Conforme citado na literatura, a análise fractal permite identificar as regionais propicias para ocorrência de eventos extremos. Dentre as variáveis de chuva, foi possível relacionar os fenômenos ZCAS e Frontais, atualmente conhecido como os principais fenômenos atmosféricos de escala sinótica desencadeador de chuvas persistentes e intensas nas Região Sudeste.

A intensidade da chuva durante uma tempestade específica, ou período mais curto, é vital para estudo dos profissionais preocupados com a previsão e prevenção de enchentes, bem como os especialistas que lidam com a erosão do solo.

Os resultados da espacialização pluviométrica da dimensão fractal demostraram semelhanças regionais com o Zoneamento Climático de Minas Gerais, principalmente nos limiares menores. A metodologia adotada permitiu delimitar e caracterizar as faixas de precipitação em diferentes períodos e limiares para uma melhor compreensão do sistema.

A distribuição da chuva não apresenta a invariância escalar temporal em relação aos segmentos determinados. Cada setor deste segmento possui um mecanismo controlante associado à dinâmica da atmosfera em cada escala temporal. Isto explica a variabilidade da frequência em relação aos segmentos e suas distorções em diferentes períodos de chuvas. Apesar da dimensão fractal não explicar a origem da precipitação, ela pode ditar o ritmo pluviométrico como estudado por Christofoletti (2007); demostrando a sua aplicabilidade no campo da Climatologia.

Para melhor acurácia da técnica, se sugere a aplicação em séries mais longas que contemplem mais períodos irregulares de precipitação, acrescentando outras variáveis como temperatura, umidade, topografia, etc. Também se pode aprofundar na questão do tempo, ao invés de dados diários de precipitação, trabalhar com dados horários, permitindo assim estudar as oscilações temporais ao decorrer de um dia.

\section{REFERÊNCIAS}

ABREU, M. L. Climatologia da estação chuvosa de Minas Gerais: de Nimer (1977) à zona de convergência do atlântico sul. Revista GEONOMOS, IGC - UFMG, 6 (2): 17-22, 2008.

AGRITEMPO - Sistema de Monitoramento Agrometeorológico. Disponível em: https://www.agritempo.gov.br/agritempo/jsp/Es tacao/index.jsp?siglaUF=MG. Acesso em: 22 set. 2016.

AYOADE, J. O. Introdução à climatologia para os trópicos. Tradução de M.J. Z. Santos. 10. ed. Rio de Janeiro: Bertrand Brasil, 2004. 332 p.

BARRY, R. G.; CHORLEY, R. J. Atmosfera, Tempo e

Clima. 9. Ed. Porto Alegre: Bookman, 2013.

CAPRA, F. A. A teia da vida. São Paulo: Cultrix, 1996.

CAVALCANTI, I. F. A.; (Org.). Tempo e clima no Brasil. São Paulo: Oficina de Textos, 2009.

CHORLEY, R. J. Geomorphology and general systems theory. U.S. Geological Survey, 


\section{ANÁLISE MULTIFRACTUAL DA VARIABILIDADE ESPACIAL PLUVIOMÉTRICA NO ESTADO DE MINAS GERAIS}

Profesional Paper 500-B, 1962. Tradução em Notícias Geomorfológica, 11 (21): pp. 3-22, 1971. CHRISTOFOLETTI, A. Análise de Sistemas em Geografia. São Paulo: Hucitec, 1979.

Modelagem de Sistemas

Ambientais. São Paulo: Edgard Blucher, 1999.

CHRISTOFOLETTI, A. L. H. Análise fractal e multifractal da estrutura de estações chuvosas em localidades do Estado de São Paulo. Tese (doutorado em Geociências e Meio Ambiente) Instituto de Geociências e Ciências Exatas, Universidade Estadual Paulista, Rio Claro, 1997.

Explanatory circular on the WMO Quality Management. Guide to Meteorological Instruments and Methods of Observation (WMONo. 488).

GUERRINI, I. A. Caos e fractais em Física Aplicada. Botucatu: IB/Unesp, 1996.

GLEICK, J. Caos: a criação de uma nova ciência. Rio de Janeiro: Campus, 1990.

HUGGET, R. J. Earth Surface Systems. Berlim: Springer Verlag, 1985.

LAM, N. S. N.; DE COLA, L. Fractals in Geography. New Jersey: PTR Prentice-Hall, 1993.

LIMBERGER, L. Abordagem sistêmica e complexidade na geografia. UNESP: Revista Geografia - V.15, n.2, jul./dez., 2006. Disponível em http://www.uel.br/revistas/geografia.

LORENZ, E. N. A essência do caos. Tradução de Cláudia Bentes David. Brasília: Editora Universidade de Brasília, 1996.

LOVEJOY, S., SCHETZER, D. and TSONIS, A.A., 1987. Functional box-counting and multiple elliptical dimensions of rain. Science, 235: 10361038.

LUCAS, T. P. B. Chuva Persistente e Ação da Zona de Convergência do Atlântico Sul na Região Metropolitana - Instituto de Geociências, Departamento de Geografia da UFMG, Belo Horizonte, 2007.

MANDELBROT, B. B. The fractal geometry of nature. New York: W. H. Freeman and Company, 1983.

MAYER, L., Fractal characteristics of desert storm sequences and implications for geomorphic studies. In: R.S. Snow and L. Mayer (Editors),
Fractals in Geomorphology. Geomorphology, 1992, 5:167-183.

MELLO, C. R.; VIOLA, M. R.; Mapeamento de chuvas intensas no Estado de Minas Gerais. Revista Bras. Ci. Solo, 37-44, 2012.

MONTEIRO, C. A. F. A dinâmica climática e as chuvas no estado de São Paulo. (Estudo geográfico sob forma de Atlas). Rio Claro, Departamento de Geografia da Faculdade de Filosofia, Ciências eLetras, 1964.

MONTEIRO, C. A. F. Análise rítmica em climatologia. Problemas da atualidade climática em São Paulo e achegas para um programa de trabalho. Climatologia, São Paulo, n.01, 1971, p.1-21.

MONTEIRO, C. A. F. A dinâmica climática e as chuvas no Estado de São Paulo: estudo geográfico sob a forma de atlas. São Paulo: IG/USP, 1973.

NIMER, E. Climatologia do Brasil. Instituto Brasileiro de Geografia e Estatística: Rio de Janeiro, 1989.

OLSSON, J.; NIEMCZYNOWICZ, J.; BERNDTSOON, R.; LARSON, M. An analysis of the rainfall time structure by box counting: some practical implications. Journal of Hydrology, Nova Iorque, v. 137, n. 1-4, p. 261-277, 1992.

PEREIRA JUNIOR, A.; CHRISTOFOLETTI, A. L. H. Análise fractal da distribuição espacial das chuvas no Estado de São Paulo. Geografia (Rio Claro), Rio Claro, SP, v.28, n.1, p.97-133, abr. 2003.

REBOITA, M.S.; GAN, M.A.; ROCHA, R.P. \& AMBRIZZI, T. Regimes de precipitação na América do Sul: uma revisão bibliográfica. R. Bras. Meteorol., 25:185-204, 2010.

SCHEIDEGGER, A. D. Teorethical Geomorphology. Berlim: Springer Verlag, 1991. SCOLFORO, J. R. S.; CARVALHO, L. M. T.; OLIVEIRA, A. D.; Zoneamento ecológicoeconômico do Estado de Minas Gerais: componentes geofísico e biótico. Lavras: Editora UFLA, 2008.

STEWART, I. Será que Deus joga dados? A nova matemática do caos. Rio de Janeiro: 1991.

STRAHLER, A. Systems theory and Physical Geography. Physical Geography: 1 (1): 1-27, 1980. 


\section{ANÁLISE MULTIFRACTUAL DA VARIABILIDADE ESPACIAL PLUVIOMÉTRICA NO ESTADO DE MINAS}

GERAIS

TARIFA, J. R. Alterações climáticas resultantes da ocupação agrícola no Brasil. Revista do Departamento de Geografia - USP, São Paulo, n. 8, p. 15-27, 1994.

THORNTHWAITE, C. W.; MATHER, J. R. The water balance. Publication in Climatology, Laboratory of Climatology, Centerton, v. 8, n.1. 1955.
VITTE, A. C.; GUERRA, A. J. T. (ORG). Reflexões sobre a geografia física no Brasil. 2. ed. Rio de Janeiro: Bertrand Brasil, 2004. C.3. P. 89-110.

ZAVATTINI, J. A.; BOIN, M. N. Climatologia geográfica: teoria e prática de pesquisa. Campinas, SP: Editora Alínea, 2013. p. 59-63 